\title{
A Methodology for the Design of Robust Rollover Prevention Controllers for Automotive Vehicles with Active Steering
}

\author{
Selim Solmaz ${ }^{\dagger}$, Martin Corless $^{\dagger}$, and Robert Shorten ${ }^{\S}$ \\ ${ }^{\dagger}$ Hamilton Institute, National University of Ireland-Maynooth, Co. Kildare, Ireland \\ ¥ School of Aeronautics \& Astronautics, Purdue University-West Lafayette, IN, USA \\ (received June 2006)
}

\begin{abstract}
In this paper we present a robust controller design methodology for vehicle rollover prevention utilizing active steering. Control design is based on keeping the magnitude of the vehicle load transfer ratio (LTR) below a certain level in the presence of driver steering inputs; we also develop an exact expression for LTR. The proposed controllers have a proportional-integral structure whose gain matrices are obtained using the results of Pancake, Corless and Brockman. These controllers reduce the transient magnitude of the LTR while maintaining the steady state steering response of the vehicle. The controllers can be designed to be robust with respect to vehicle parameters such as speed and centre of gravity height. We also provide a modification to the controllers so that they only activate when the potential for rollover is significant. Numerical simulations demonstrate the efficacy of our approach and the resulting controllers.
\end{abstract}

\section{Outline of the Paper}

1. Introduction

2. Related work

3. Vehicle modelling and $L T R_{d}$

3.1. Vehicle model

3.2. The dynamic load transfer ratio, $L T R_{d}$

3.3. Actuators sensors and parameters

4. State feedback controllers for robust disturbance attenuation

5. Rollover control design

5.1. (a) Active steering PI controller with known parameters 5.1.1. Simulations

5.2. (b) Robust control design

5.2.1. Simulations

5.2.2. Controller mode switch

6. Conclusions

7. References

A. Appendix: Iterative algorithm for robust control design

Keywords: Vehicle dynamics control; Rollover prevention; Active Steering; Robustness with respect to parameter uncertainty.

\section{Introduction}

It is well known that vehicles with a high center of gravity such as vans, trucks and the highly popular SUVs (Sport Utility Vehicles) are more prone to rollover accidents. According to the 2004 data (NTHSA, 2006), light trucks (pickups, vans and SUVs) were involved in nearly $70 \%$ of all the rollover accidents in the USA, with SUVs alone responsible for almost $35 \%$ of this total. The fact that the composition of the current automotive fleet in the U.S. consists of nearly 36\% pickups, vans and SUVs (Carlson et al., 2003), along with the recent increase in the popularity of SUVs worldwide, makes rollover an important safety problem.

† Email: selim.solmaz@nuim.ie, Fax: +353 1 7086269, Phone: +353 17086100

\$ Email: corless@ @urdue.edu, Fax: +1 765 4940307, Phone: +1 7654947411

§ Email: robert.shorten@ nuim.ie, Fax: +3531 7086269, Phone: +3531 1086100 
There are two distinct types of vehicle rollover: tripped and un-tripped rollover. Tripped rollover is usually caused by impact of the vehicle with something else (e.g. obstacles, curb etc.) resulting in the rollover incident. Driver induced un-tripped rollover can occur during typical driving situations and poses a real threat for top-heavy vehicles. Examples are excessive speed during cornering, obstacle avoidance and severe lane change maneuvers, where rollover occurs as a direct result of the wheel forces induced during these maneuvers. It is however, possible to prevent such a rollover incident by monitoring the car dynamics and applying appropriate control effort ahead of time. Therefore there is a need to develop driver assistance technologies which would be transparent to the driver during normal driving conditions, but which act when needed to recover handling of the vehicle during extreme maneuvers (Carlson et al., 2003).

In this paper we present a robust rollover prevention controller design methodology based on active steering. As an accurate indicator of impending rollover, we consider the vehicle Load Transfer Ratio (LTR). Vehicle wheel lift off occurs when the magnitude of this variable reaches one. We develop an exact expression for this variable taking the vehicle roll dynamics fully into account. To distinguish our expression from previous approximations of LTR in the literature, we denote it by $L T R_{d}$; these approximations usually ignored roll dynamics.

Our proposed controllers have a PI (proportional-integral) structure with two fixed gain matrices $K_{P}$ and $K_{I}$. By utilizing the integral action in the controller, we ensure that the steady state steering response of the vehicle is as expected by the driver. The gain matrices are chosen to reduce the magnitude of $L T R_{d}$ during transient behavior.

The design of the controller gain matrices is based on recent results in (Pancake et al., 2000) where they consider uncertain systems with performance outputs and subject to a bounded disturbance input. For each output $z_{j}$ they introduce a performance measure $\gamma_{j}$ which guarantees that the magnitude of the output is less than or equal to $\gamma_{j}$ times the peak value of the magnitude of the disturbance. They present a controller design procedure which can be used to minimize the performance level for one main output while keeping the performance levels for the other outputs below some prespecified levels. In addition the controllers in (Pancake et al., 2000) are robust in the sense that they ensure performance in the presence of any allowable uncertainty which was taken into account in the control design. In applying the results from (Pancake et al., 2000), we consider the driver steering input as a disturbance input. Since we wish to keep the magnitude of $L T R_{d}$ less than one, we view this as the main performance output. To limit the amount of control effort, we choose the control input as an additional performance output. Many control designs in the literature are based on keeping the root mean square of a performance output small. However, we consider it more important to utilize a controller which is designed to keep the peak magnitude of $L T R_{d}$ small rather than its rms value.

We initially consider control design for fixed vehicle parameters and illustrate the efficacy of our approach with some numerical simulations using typical data for a compact car. We then design a fixed robust controller which is effective for a range of vehicle speeds and vehicle CG (centre of gravity) heights. The efficacy of this controller is illustrated by simulating the vehicle with different CG heights and with varying speeds. Finally, we propose a modification to our controllers so that they only activate when the potential for rollover is significant. This modification prevents the controllers from activating in non-critical situations and possibly annoying the driver.

\section{Related work}

Rollover prevention is a topical area of research in the automotive industry and several studies have recently been published. Relevant publications include that of Palkovics et al. (1999), where they proposed the ROP (Roll-Over Prevention) system for use in commercial trucks making use of lateral acceleration measurement as well as the wheel slip difference on the two sides of the axles to predict tire lift-off prior to rollover. They utilized full braking action through EBS (Electronic Brake System) in the event that tire lift-off is detected, which in turn reduces vehicle speed to eliminate the rollover threat. In a similar implementation, Wielenga (1999) suggested the ARB (Anti Roll Braking) system utilizing braking of the individual front wheel outside the turn or the full front axle instead of the full braking action. The suggested control system is based on lateral acceleration thresholds and/or tire lift-off sensors in the form of simple contact switches. Again making use of differential braking actuators, Chen et al. (2001) suggested utilizing an estimated TTR (Time To Rollover) metric as an early indicator for the rollover threat. When TTR is less than a certain preset threshold value for the particular vehicle under interest, they utilized differential breaking to prevent rollover. Ackermann et al. (1998), and Odenthal et al. (1999) proposed a robust active steering controller, as well as a combination of active steering and emergency braking controllers. 
They utilized an active steering controller based on roll rate measurement. They also suggested the use of a static Load Transfer Ratio $\left(L T R_{S}\right)$ which is based on lateral acceleration measurement; this was utilized as a criterion to activate the emergency steering and braking controllers. Carlson et al. (2003) made use of sideslip, yaw rate, roll angle and roll rate measurements based on GPS aided INS (Inertial Navigation System) along with steer by wire and differential braking actuators to limit excessive roll angle during dangerous maneuvers. They based their controller design on MPC (Model Predictive Control).

\section{Vehicle modelling and $L T R_{d}$}

In this section we introduce the model that we use for controller design. We also define the rollover detection criterion $L T R_{d}$ and present the assumptions on the sensors and actuators used in the design.

\subsection{Vehicle model}

In order to capture the salient features of vehicle rollover and for controller design purposes, we utilize the well known linearized vehicle model commonly referred as the single-track model (or bicycle model) with a roll degree of freedom; this is illustrated in Figure 1. This specific model or its variations are widely used in vehicle dynamics control applications (see for example Carlson et al. (2003), Takano et al. (2001), Ackermann et al. (1998), Odenthal et al. (1999), Chen et al. (2001), Hac et al. (2004), Kiencke et al. (2000)). In this linear model the steering angle $\delta$, the roll angle $\phi$, and the vehicle sideslip angle $\beta$ are all assumed to be small. We further assume that all the vehicle mass is sprung, which implies insignificant wheel and suspension weights. Also
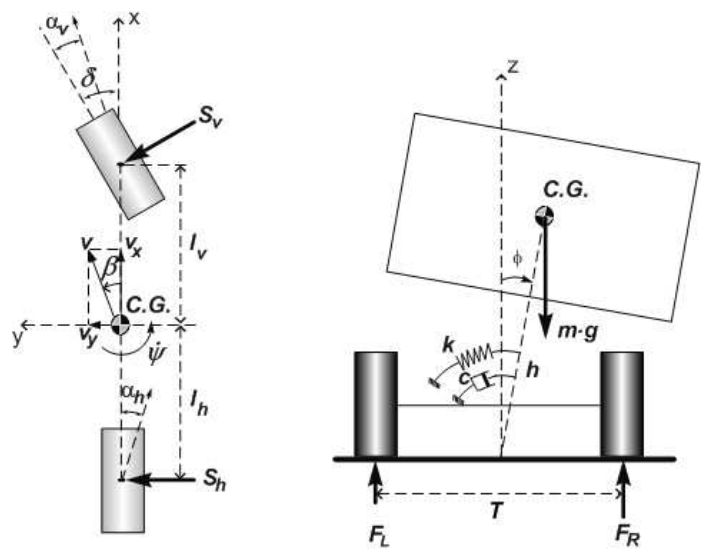

Figure 1. Single track model with roll degree of freedom.

the lateral forces on the front and rear tires, denoted by $S_{v}$ and $S_{h}$, respectively, are represented as linear functions of the tire slip angles $\alpha_{v}$ and $\alpha_{h}$, that is, $S_{v}=C_{v} \alpha_{v}$ and $S_{h}=C_{h} \alpha_{h}$, where $C_{v}$ and $C_{h}$ are the front and rear tire stiffness parameters respectively. The assumptions of small angles and linear tire forces are probably an over simplification of the nonlinear vehicle behavior at the rollover limit, yet these provide a good balance between capturing the salient features of vehicle behavior while keeping the complexity at a manageable level. In order to simplify the model description, we further define the following auxiliary variables

$$
\begin{aligned}
& \sigma \triangleq C_{v}+C_{h}, \\
& \rho \triangleq C_{h} l_{h}-C_{v} l_{v}, \\
& \kappa \triangleq C_{v} l_{v}^{2}+C_{h} l_{h}^{2},
\end{aligned}
$$

where the lengths $l_{v}$ and $l_{h}$ are defined in Figure 1. For simplicity, it is assumed that the sprung mass rolls about a horizontal roll axis which is along the centerline of the track and at ground level. Using the parallel axis theorem of 


\begin{tabular}{cll} 
Table 1. & Model Parameters and their definitions & \\
\hline Parameter & Description & Unit \\
\hline$m$ & vehicle mass & {$[\mathrm{kg}]$} \\
$v$ & vehicle speed & {$[\mathrm{m} / \mathrm{s}]$} \\
$\delta$ & steering angle & {$[\mathrm{rad}]$} \\
$J_{x x}$ & roll moment of inertia of the sprung mass measured at the CG & {$\left[\mathrm{kg} \cdot \mathrm{m}^{2}\right]$} \\
$J_{z z}$ & yaw moment of inertia of the chassis measured at the CG & {$\left[\mathrm{kg} \cdot \mathrm{m}^{2}\right]$} \\
$l_{v}$ & longitudinal CG position measured w.r.t. the front axle & {$[\mathrm{m}]$} \\
$l_{h}$ & longitudinal CG position measured w.r.t. the rear axle & {$[\mathrm{m}]$} \\
$h$ & CG height measured over the ground & {$[\mathrm{m}]$} \\
$c$ & suspension damping coefficient & {$\left[\mathrm{kg} \cdot \mathrm{m}^{2} / \mathrm{s}\right]$} \\
$k$ & suspension spring stiffness & {$\left[\mathrm{kg} \cdot \mathrm{m}^{2} / \mathrm{s}^{2}\right]$} \\
$C_{v}$ & linear tire stiffness coefficient for the front tire & {$[\mathrm{N} / \mathrm{rad}]$} \\
$C_{h}$ & linear tire stiffness coefficient for the rear tire & {$[\mathrm{N} / \mathrm{rad}]$} \\
\hline
\end{tabular}

mechanics, $J_{x_{e q}}$, the moment of inertia of the vehicle about the assumed roll axis, is given by

$$
J_{x_{e q}}=J_{x x}+m h^{2}
$$

where $h$ is the distance between the center of gravity (CG) and the assumed roll axis and $J_{x x}$ is the moment of inertia of the vehicle about the roll axis through the CG. We introduce the state vector $\xi=\left[\begin{array}{llll}v_{y} & \dot{\psi} & \dot{\phi} & \phi\end{array}\right]^{T}$, where

$v_{y}$ : lateral velocity of the vehicle,

$\dot{\psi}$ : yaw rate of the undercarriage,

$\dot{\phi}$ : roll rate of the sprung mass about the roll axis,

$\phi$ : roll angle of the sprung mass about the roll axis.

The linearized equations of motion corresponding to this model are as follows

$$
\dot{\xi}=\tilde{A} \xi+\tilde{B} \delta
$$

with

$$
\tilde{A}=\left[\begin{array}{cccc}
-\frac{\sigma J_{x e q}}{m v J_{x x}} & \frac{\rho J_{x e q}}{m v J_{x x}}-v & -\frac{h c}{J_{x x}} & \frac{h(m g h-k)}{J_{x x}} \\
\frac{\rho}{J_{z z} v} & -\frac{\kappa}{J_{z z} v} & 0 & 0 \\
-\frac{h \sigma}{v J_{x x}} & \frac{h \rho}{v J_{x x}} & -\frac{c}{J_{x x}} & \frac{m g h-k}{J_{x x}} \\
0 & 0 & 1 & 0
\end{array}\right], \quad \tilde{B}=\left[\begin{array}{c}
\frac{C_{v} J_{x e q}}{m J_{x x}} \\
\frac{C_{v} l_{v}}{J_{z z}} \\
\frac{h C_{v}}{J_{x x}} \\
0
\end{array}\right] .
$$

Further definitions of the parameters appearing in (3) are given in Table 1. Also see Kiencke et al. (2000) for a detailed description and derivation of this vehicle model.

\subsection{The dynamic load transfer ratio, $L T R_{d}$}

Traditionally, as discussed in the related work section, some estimate of the vehicle load transfer ratio has been used as a basis for the design of rollover prevention systems. The load transfer ratio (Odenthal et al., 1999; Kamnik et $a l ., 2003$ ) can be simply defined as the load (i.e., vertical force) difference between the right and left wheels of the vehicle, normalized by the total load (i.e., the weight of the car). In other words,

$$
\text { Load transfer ratio }=\frac{\text { Load on right tires }- \text { Load on left tires }}{\text { Total weight }}
$$


Clearly, this quantity varies between -1 and 1 , and for a perfectly symmetric vehicle that is driving in a straight line, it is zero. The extrema are reached in the case of a wheel lift-off on one side of the vehicle, in which case the load transfer ratio is 1 or -1 depending on the side that lifts off. If roll dynamics are ignored, it is easily shown (Odenthal et al., 1999) that the corresponding load transfer ratio (which we denote by $L T R_{S}$ ) is approximated by

$$
L T R_{s}=\frac{2 a_{y} h}{g T}
$$

where $a_{y}$ is the lateral acceleration of the CG and $T$ is the vehicle track width.

Note that rollover estimation based upon (5) is not sufficient to detect the transient phase of rollover (due to the fact that it is derived ignoring roll dynamics). In (Solmaz et al., 2006) we obtain an exact expression for the vehicle load transfer ratio which does not ignore roll dynamics; we denote this by $L T R_{d}$. To aid exposition we repeat the derivation here. Recall that we assumed the unsprung mass weight to be insignificant and the main body of the vehicle rolls about an axis along the centerline of the track at the ground level. We can write a torque balance for the unsprung mass about the assumed roll axis in terms of the suspension torques and the vertical wheel forces as follows:

$$
-F_{R} \frac{T}{2}+F_{L} \frac{T}{2}+k \phi+c \dot{\phi}=0
$$

Now substituting the definition of load transfer from (4) and rearranging yields the following expression for $L T R_{d}$ :

$$
L T R_{d}=\frac{2}{m g T}(c \dot{\phi}+k \phi) \text {. }
$$

In terms of the state, $L T R_{d}$ can be represented by the following relationship

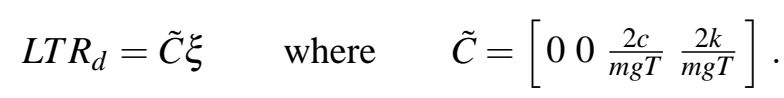

We now provide a brief description of the actuators to be used in implementing our proposed active steering controllers.

\subsection{Actuators, sensors and parameters}

We are interested in robust control design based on active steering actuators. There are two types of active steering methods: full steer-by-wire and mechatronic-angle-superposition types. Steer-by-wire actuators do not contain a physical steering column between the steering wheel and the wheels; the steering torque is generated solely by a servo motor based on the driver steering command. This enables steer-by-wire actuators to be flexible and suitable for various vehicle dynamics control applications. However, stringent safety requirements on such systems prevent them from entering today's series-production vehicles. Mechatronic-angle-superposition type active steering actuators however have been recently introduced to the market. They contain a physical steering column and act cooperatively with the driver, while they permit various functions such as speed dependent steering ratio modification, and active response to mild environmental disturbances. It is plausible that active steering actuators will become an industry standard in the near future, due to their capability of directly and most efficiently affecting the lateral dynamics of the car. Active steering based lateral control methods can be perfectly transparent to the driver and they are likely to cause the least interference with the driver intent unlike the control approaches based on differential braking and active suspension. Moreover, the use of active steering actuators do not result in a significant velocity loss, therefore they are likely to enter the market initially for the high performance vehicle segment.

In this paper we assume mechatronic-angle-superposition type steering actuators with access to full state information. Although such active steering actuators require inputs from the driver, for the sake of keeping the discussion as simple as possible, in this paper we assume no internal actuator dynamics or delays that might arise from driver interactions. It is however possible to account for the effects of these in the controller de- 
sign. Also our results can easily be extended to the case of steer-by-wire actuators where driver interactions are of less importance.

We also assume in this paper that all the model parameters $m, J_{x x}, J_{z z}, l_{v}, l_{h}, C_{v}, C_{h}, k, h, c$ are known. This is an unrealistic assumption: yet our control design is easily extended to account for uncertainty in these parameters which we demonstrate by designing our controllers to be robust with respect to uncertainties in vehicle speed $v$ and center of gravity height $h$. As a side note, although we assumed all the vehicle model parameters to be known, it is possible to estimate some of these that are fixed (but unknown) using the sensor information available for the control design suggested here; this however is outside the scope of this work (see Akar et al., 2006).

\section{State feedback controllers for robust disturbance attenuation}

In a later section, we will utilize the results obtained by Pancake, Corless and Brockman (Pancake et al., 2000, 2006) to design controller gain matrices. (Pancake et al., 2000, 2006) consider uncertain systems of the form

$$
\begin{aligned}
\dot{x} & =A(\theta) x+B(\theta) \omega+B_{u}(\theta) u \\
z_{j} & =C_{j}(\theta) x+D_{j}(\theta) \omega+D_{j u}(\theta) u,
\end{aligned}
$$

where $\theta$ is some parameter vector (which can be time and state dependent) that captures the plant nonlinearity/uncertainty. The vector vector $x(t)$ is the state at time $t$ and $\omega(t)$ is a bounded disturbance input while $u(t)$ is the control input and $z_{1}(t), \ldots, z_{r}(t)$ are the performance outputs. For each output $z_{j}$ (Pancake et al., 2000, 2006) introduce a measure of performance measure $\gamma_{j}$ which guarantees that the magnitude of that output is less than or equal to $\gamma_{j}$ times the peak value of the magnitude of the disturbance. They present a controller design strategy which can be used to minimize the performance level for one main output while keeping the performance levels for the other outputs below some prespecified levels. In addition the controllers in (Pancake et al., 2000) are robust in the sense that they ensure performance in the presence of any allowable uncertainty which was taken into account in the control design. The uncertainty in the plant is required to satisfy the following condition.

Assumption 4.1 For each $\theta$ and $j=1, \ldots, r$, the matrix

$$
\left[A(\theta) B(\theta) B_{u}(\theta) C_{j}(\theta) D_{j}(\theta) D_{j u}(\theta)\right]
$$

can be written as a convex combination of a finite number of matrices (called vertex matrices)

$$
\left[\begin{array}{llllll}
A_{1} & B_{1} & B_{u_{1}} & C_{j_{1}} & D_{j_{1}} & D_{j u_{1}}
\end{array}\right], \ldots,\left[A_{N} B_{N} B_{u_{N}} C_{j_{N}} D_{j_{N}} D_{j u_{N}}\right]
$$

Remark 1 Suppose that each of the matrices $A(\theta), B(\theta), B_{u}(\theta), C_{j}(\theta), D_{j}(\theta), D_{j u}(\theta)$ depend in a multi-affine fashion on the components of the $M$-vector $\theta$ and each element of $\theta$ is bounded, that is,

$$
\underline{\theta}_{k} \leq \theta_{k} \leq \bar{\theta}_{k} \quad \text { for } \quad k=1, \ldots, M .
$$

Then, for all $\theta$, the matrix in (11) can be expressed as a convex combination of the $2^{M}$ matrices corresponding to the extreme values of the components of $\theta$; these vertex matrices are given by

$$
\left[A(\theta) B(\theta) B_{u}(\theta) C_{j}(\theta) D_{j}(\theta) D_{j u}(\theta)\right] \quad \text { where } \quad \theta_{k}=\underline{\theta}_{k} \text { or } \bar{\theta}_{k} \text { for } k=1, \ldots, M
$$

The following result from (Pancake et al., 2000, 2006) is useful in designing our rollover prevention controllers.

THEOREM 4.2 Consider a nonlinear/uncertain system described by (9)-(10) and satisfying Assumption 4.1. Suppose that there exist a matrix $S=S^{T}>0$, a matrix $L$ and scalars $\beta_{1}, \ldots \beta_{N}>0$ and $\mu_{0}, \mu_{1 j}, \mu_{2 j} \geq 0, j=1, \ldots, r$, 
such that the following matrix inequalities hold

$$
\begin{gathered}
{\left[\begin{array}{rr}
\beta_{i}\left(S A_{i}^{T}+A_{i} S+L^{T} B_{u_{i}}^{T}+B_{u_{i}} L\right)+S & \beta_{i} B_{i} \\
\beta_{i} B_{i}^{T} & -\mu_{0} I
\end{array}\right] \leq 0} \\
{\left[\begin{array}{ccr}
-\mu_{1 j} S & 0 & S C_{j_{i}}^{T}+L^{T} D_{j u_{i}}^{T} \\
0 & -\mu_{2 j} I & D_{j_{i}}^{T} \\
C_{j_{i}} S+D_{j u_{i}} L & D_{j_{i}} & -I
\end{array}\right] \leq 0}
\end{gathered}
$$

for all $i=1, \ldots, N$ and $j=1, \ldots, r$. Then the controller

$$
u=K x \quad \text { with } \quad K=L S^{-1}
$$

results in a closed loop nonlinear/uncertain system which has the following properties.

(a) The undisturbed system $(\omega=0)$ is globally exponentially stable, that is, all state trajectories decay exponentially.

(b) If the disturbance input is bounded, that is, $\|\omega(t)\| \leq \rho_{\omega}$ for all t then, for zero initial state, the performance outputs $z_{1}, \ldots, z_{r}$ of the closed loop system are bounded and satisfy

$$
\left\|z_{j}(t)\right\| \leq \gamma_{j} \rho_{\omega}
$$

for all $t$ where

$$
\gamma_{j}=\sqrt{\mu_{0} \mu_{1 j}+\mu_{2 j}}
$$

The scalars $\gamma_{1}, \ldots \gamma_{r}$ are called levels of performance and can be regarded as measures of the ability of the closed loop system to attenuate the effect of the disturbance input on the performance outputs; a smaller $\gamma_{j}$ means better performance in the sense of increased attenuation. For a proof of the theorem, see (Pancake et al., 2006).

Remark 2 Consider the situation in which the matrices $D_{j_{1}}, \ldots, D_{j_{N}}$ are all zero for some performance output $z_{j}$. Then, for each $i$, inequality (14) is satisfied for some $\mu_{2 j} \geq 0$ if and only if it is satisfied with $\mu_{2 j}=0$. Hence, if $D_{j_{1}}, \ldots, D_{j_{N}}$ are all zero, inequality (14) can be replaced with

$$
\left[\begin{array}{cc}
-\mu_{1 j} S & S C_{j_{i}}^{T}+L^{T} D_{j u_{i}}^{T} \\
C_{j_{i}} S+D_{j u_{i}} L & -I
\end{array}\right] \leq 0
$$

In this case,

$$
\gamma_{j}=\sqrt{\mu_{0} \mu_{1 j}}
$$

Also, using Schur complements, one can show that the above inequality is equivalent to the following inequality which is linear in the variables $S$ and $\mu_{1 j}$.

$$
\left[\begin{array}{cc}
-S & S C_{j_{i}}^{T}+L^{T} D_{j u_{i}}^{T} \\
C_{j_{i}} S+D_{j u_{i}} L & -\mu_{1 j} I
\end{array}\right] \leq 0
$$

Remark 3 Consider the closed loop system subject to a fixed bounded disturbance $\omega$ which satisfies $\|\omega(t)\| \leq \rho_{\omega}$, let

$$
V(x)=x^{T} P x
$$


and consider the bounded ellipsoid in state space defined by

$$
\mathscr{E}\left(\rho_{\omega}\right)=\left\{x \in \mathbb{R}^{n}: V(x) \leq \mu_{0} \rho_{\omega}^{2}\right\}
$$

The inequalities in (13) guarantee that whenever a state trajectory is outside of the ellipsoid the time rate change of the Lyapunov function $V$ is negative. From this one can show that the ellipsoid is both invariant and attractive. Attractive means that all state trajectories converge to the ellipsoid with increasing time. Invariance means that if a state trajectory starts in the ellipsoid, it remains there forever; in particular, if a trajectory starts at the origin, it will always be contained in the ellipsoid.

The inequalities in (14) guarantee that each performance output $z_{j}$ satisfies

$$
\left\|z_{j}(t)\right\|^{2} \leq \mu_{1 j} V(x(t))+\mu_{2 j} \omega(t)^{2} .
$$

Hence, if a trajectory starts within the ellipsoid, it must satisfy $\left\|z_{j}(t)\right\| \leq \gamma_{j} \rho_{\omega}$ for all $t$. Otherwise, $\left\|z_{j}(t)\right\|$ is "eventually bounded" by $\gamma_{j} \rho_{\omega}$.

\section{Rollover control design}

We now apply the results described in the previous section to the rollover prevention problem. We first present a design under the assumption that the plant parameters are known and fixed (Part a). We then extend our design to cope with plant parameter uncertainties (Part b). Finally, we further refine our design to incorporate a mode switch to deactivate the controller in situations when there is no rollover danger (Part c).

\section{1 (a) Active steering PI controller with known plant parameters}

Our objective here is to superimpose an active steering control input $u=\delta_{c}$ on the driver steering input $\delta_{d}$ to prevent rollover. Thus, the total steering input $\delta$ to the vehicle consists of two parts and is given by

$$
\delta=\delta_{d}+u
$$

The driver input $\delta_{d}$ will be regarded as a disturbance input $\omega$. Recalling model (3), our system is now described by

$$
\dot{\xi}=\tilde{A} \xi+\tilde{B} \omega+\tilde{B} u
$$

where $\xi(t) \in \mathbb{R}^{4}$ is the state at time $t \in \mathbb{R}, u(t)$ is a scalar control input and $\omega(t)$ is a scalar disturbance input. The matrices $\tilde{A}$ and $\tilde{B}$ are fixed and are as described as in (3).

We propose a proportional-integral (PI) type state feedback controller of the form

$$
u=K_{P} \xi+K_{I} \xi_{I}
$$

where the integrator state $\xi_{I}$ is the integral of the yaw rate tracking error:

$$
\dot{\xi}_{I}=\dot{\psi}-\dot{\psi}_{d}, \quad \xi_{I}(0)=0 .
$$

The reference yaw rate $\dot{\psi}_{d}$ is given by

$$
\dot{\psi}_{d}=\alpha \delta_{d},
$$

for a constant gain $\alpha$. Although this is a major simplification of the reference driver intent, we chose this linear expression for the sake of simplicity. The resulting control structure is depicted in Figure 2 below. 
Comment : The purpose of utilizing the integral action in the controller is to guarantee that when driver input $\delta_{d}$ is constant, the corresponding steady state yaw rate is given by $\dot{\psi}=\dot{\psi}_{d}=\alpha \delta_{d}$. This yaw rate will be large for large $\delta_{d}$ and will result in a large steady state value of $L T R_{d}$. To avoid this one could saturate $\dot{\psi}_{d}$ at a certain value such that, in steady state, $\|L T R\|$ stays below 1 , regardless of the driver input.

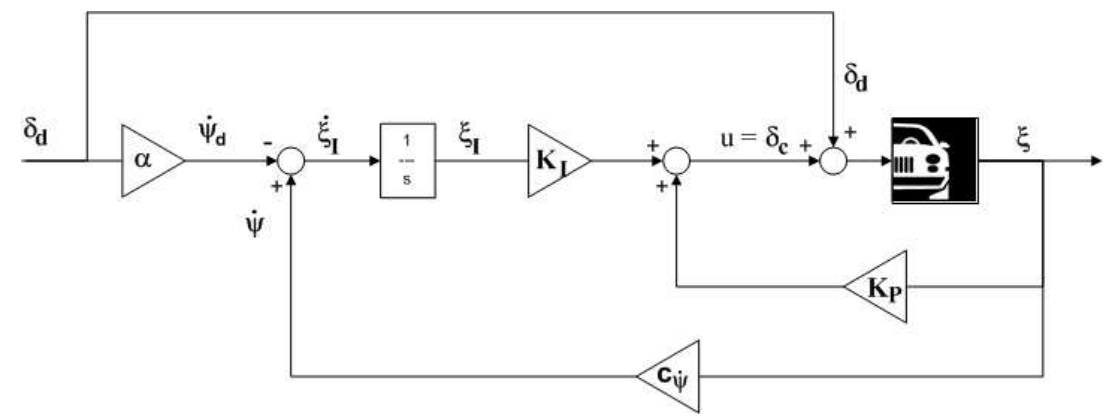

Figure 2. Flow diagram of the PI active steering controller.

We want the controller to keep the magnitude of $L T R_{d}$ small during transients with reasonable control effort. In view of this, we introduce the following two performance outputs:

$$
\begin{aligned}
& z_{1}=L T R_{d}=\tilde{C} \xi \\
& z_{2}=u,
\end{aligned}
$$

where $\tilde{C}$ is given in (7). Augmenting the vehicle dynamics with the integrator dynamics and introducing the augmented state $x=\left[\begin{array}{ll}\xi^{T} & \xi_{I}\end{array}\right]^{T}$ results in the following system description:

$$
\begin{aligned}
\dot{x} & =A x+B \omega+B_{u} u \\
z_{1} & =C_{1} x \\
z_{2} & =D_{2 u} u
\end{aligned}
$$

where

$$
A=\left[\begin{array}{cc}
\tilde{A} & 0 \\
c_{\dot{\psi}} & 0
\end{array}\right], \quad B=\left[\begin{array}{r}
\tilde{B} \\
-\alpha
\end{array}\right], \quad B_{u}=\left[\begin{array}{c}
\tilde{B} \\
0
\end{array}\right], \quad C_{1}=\left[\begin{array}{ll}
\tilde{C} & 0
\end{array}\right], \quad D_{2 u}=1
$$

and $c_{\dot{\psi}}=\left[\begin{array}{llll}0 & 1 & 0 & 0\end{array}\right]$. Also, a proposed controller (26) can be described by $u=K x$ where

$$
K=\left[K_{P} K_{I}\right] .
$$

In view of our original control objectives, we will use the results of Theorem 4.2 to obtain a gain matrix $K$ which minimizes the level of performance $\gamma_{1}$ for $z_{1}$ while keeping the level of performance $\gamma_{2}$ for $z_{2}$ below some prespecified level $\bar{\gamma}_{2}$.

5.1.1 Simulations. The model parameters used here are given in Table 2. They are typical for a compact car. The steering ratio was assumed to be $1: 18$. In using Theorem 4.2 to obtain a gain matrix $K$ which minimizes the level of performance $\gamma_{1}$ for $z_{1}$ subject to a specified level of performance $\gamma_{2}$ for $z_{2}$, we used a simplified version of the iterative solution algorithm described in the Appendix with $N=1$.

In the numerical simulations presented here, we simulated an obstacle avoidance maneuver that is commonly known as the elk-test. The maneuver takes place at a speed of $v=140 \mathrm{~km} / \mathrm{h}$ and with a peak steering magnitude of $100^{\circ}$. The results of the simulations are presented in Figure 3, which demonstrates the effectiveness of the 
Table 2. Fixed model parameters

\begin{tabular}{cll}
\hline parameter & value & unit \\
\hline$m$ & 1224.1 & {$[\mathrm{~kg}]$} \\
$J_{x x}$ & 362 & {$\left[\mathrm{~kg} \cdot \mathrm{m}^{2}\right]$} \\
$J_{z z}$ & 1279 & {$\left[\mathrm{~kg} \cdot \mathrm{m}^{2}\right]$} \\
$l_{v}$ & 1.102 & {$[\mathrm{~m}]$} \\
$l_{h}$ & 1.254 & {$[\mathrm{~m}]$} \\
$T$ & 1.51 & {$[\mathrm{~m}]$} \\
$h$ & 0.375 & {$[\mathrm{~m}]$} \\
$c$ & 4000 & {$\left[\mathrm{~kg} \cdot \mathrm{m}^{2} / \mathrm{s}\right]$} \\
$k$ & 36075 & {$\left[\mathrm{~kg} \cdot \mathrm{m}^{2} / \mathrm{s}^{2}\right]$} \\
$C_{v}$ & 90240 & {$[\mathrm{~N} / \mathrm{rad}]$} \\
$C_{h}$ & 180000 & {$[\mathrm{~N} / \mathrm{rad}]$} \\
\hline
\end{tabular}

controller in preventing rollover in this dangerous maneuver by keeping the magnitude of $L T R_{d}$ less than one. Notice that driver intervention of the controller as measured by the difference in roll angles of the controlled and uncontrolled vehicles show a slight difference, implying that the control action would probably be undiscernible by the driver, which is favorable and was one of our aims.
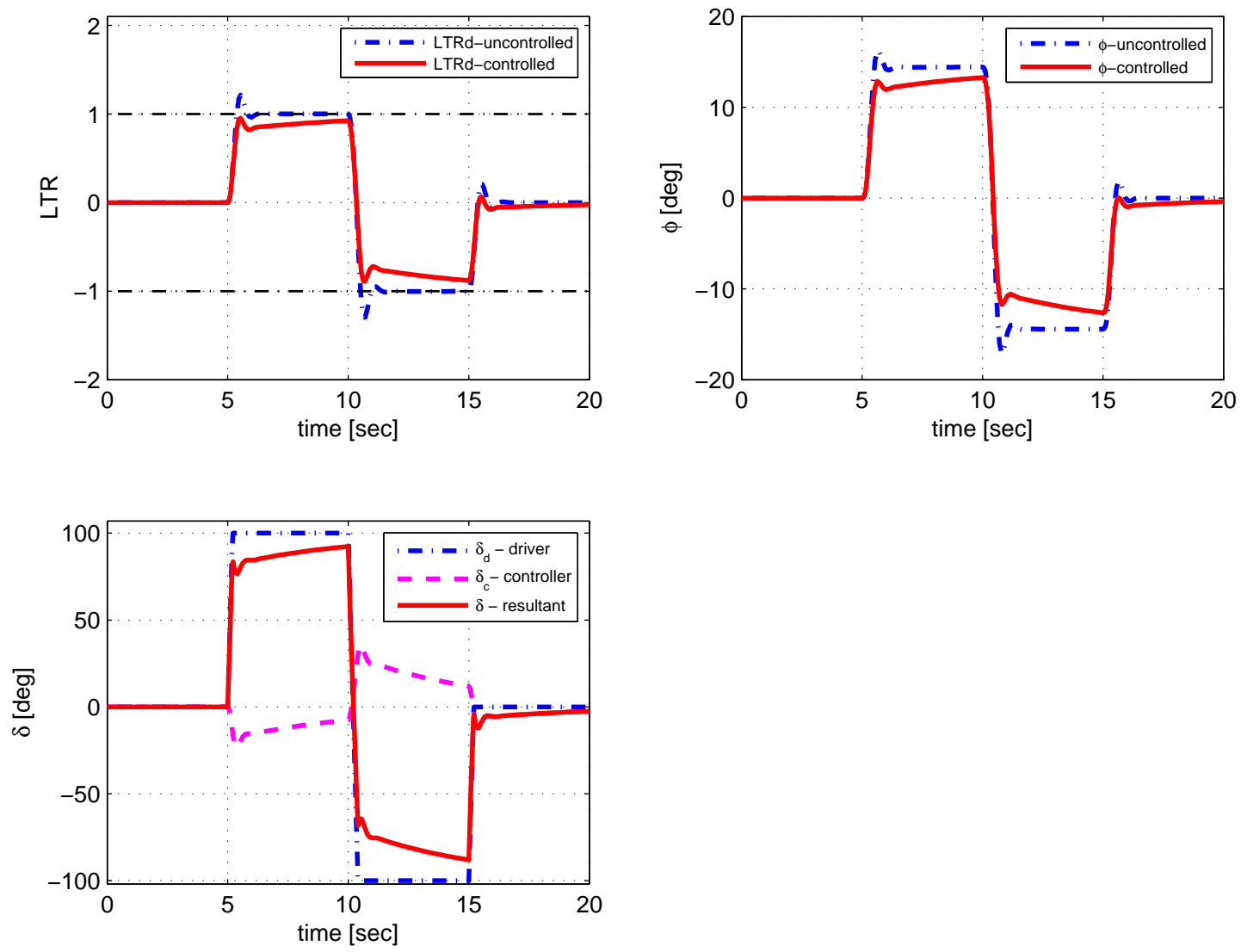

Figure 3. Comparison of the controlled (with fixed model) and uncontrolled vehicles.

It is of particular interest for us to see how the suggested controllers affect the vehicle path. To do this, we note that the coordinates $(x, y)$ of the vehicle $\mathbf{C G}$ relative to the road satisfy

$$
\begin{aligned}
& \dot{x}=v \cos (\beta+\psi), \\
& \dot{y}=v \sin (\beta+\psi),
\end{aligned}
$$


where we choose the initial coordinates $(x(0), y(0))$ to be zero. In Figure 4 the CG trajectories of the controlled and the uncontrolled vehicles are compared along with the remaining states. We observe from trajectory plots that control action causes a small divergence from the uncontrolled vehicle path during the first half of the maneuver while preventing rollover; in a real driving situation, the driver would time the second half of the maneuver based on the speed and location of the vehicle. Also similar to the roll angle variation, the remaining state plots of the controlled vehicle are close to those of the uncontrolled vehicle during the maneuver.
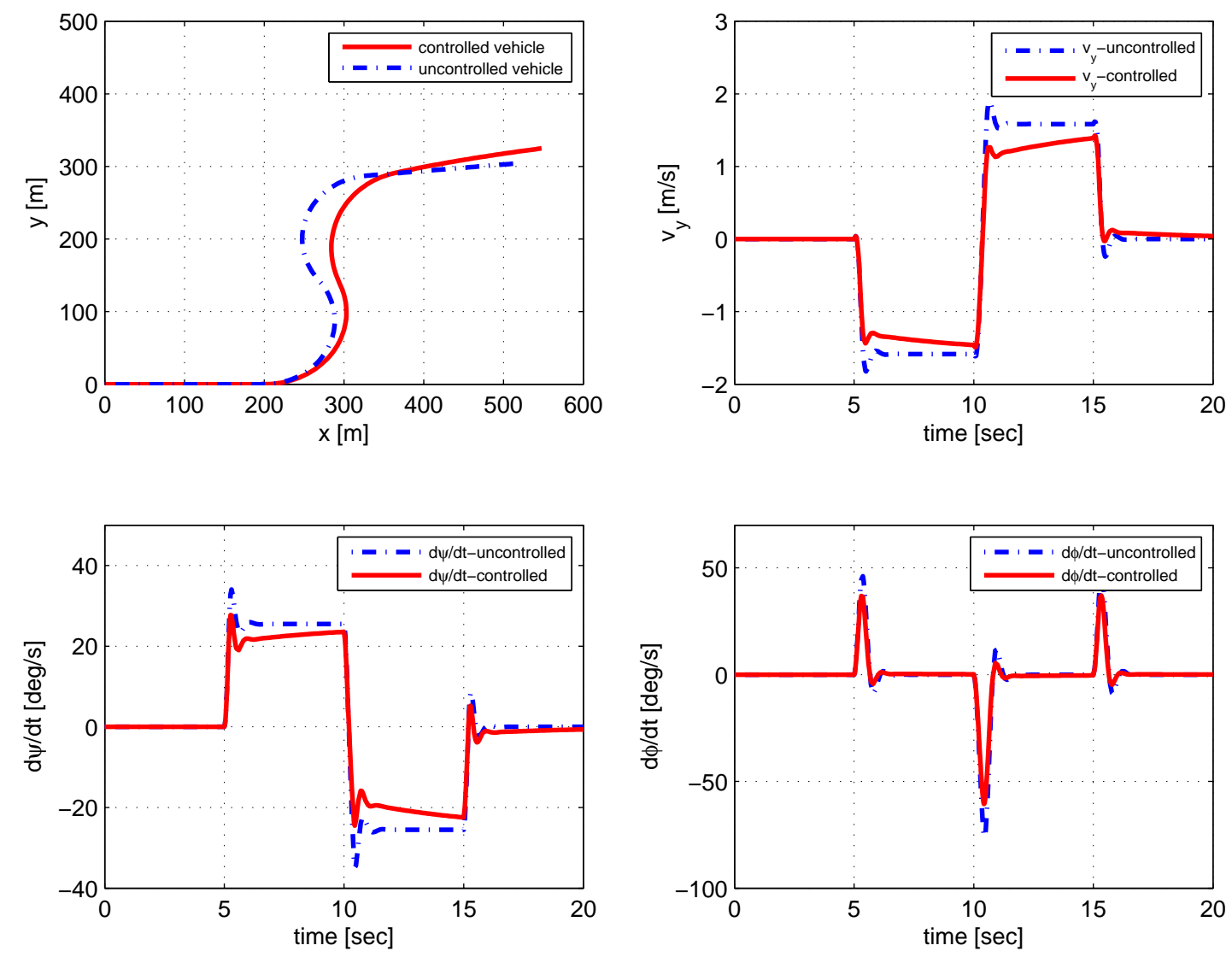

Figure 4. Comparison of the controlled (with fixed model) and uncontrolled vehicle states and trajectories.

\section{2 (b) Robust control design}

We now extend the design to cope with parameter uncertainty. Specifically, we now redesign the controller to take into account the parameter uncertainties resulting from bounded vehicle speed variations as well as CG height uncertainties by utilizing Assumption 4.1 and using Theorem 4.2.

In what follows we shall assume that the vehicle speed $v$ is bounded, that is, $\underline{v} \leq v \leq \bar{v}$, where $\underline{v}$ and $\bar{v}$ denote the lower and upper bounds on the speed, respectively. In order to represent typical freeway driving conditions we chose the speed extrema as $\underline{\mathrm{v}}=20 \mathrm{~m} / \mathrm{s}$ and $\bar{v}=40 \mathrm{~m} / \mathrm{s}$ in the numerical simulations below. We further assume that uncertain CG height $h$ belongs to the interval $[\underline{h}, \bar{h}]$, where $\underline{h}=0.2[\mathrm{~m}]$, and $\bar{h}=0.5[\mathrm{~m}]$ denote the lower and upper bounds of the uncertain CG height, respectively.

We proceed as in the previous section where we used (25) as the vehicle model for our control design and the 
matrices $\tilde{A}$ and $\tilde{B}$ are described in (3). Note that these matrices depend in a multi-affine fashion on the parameters

$$
\theta_{1}:=1 / v, \quad \theta_{2}:=v, \quad \theta_{3}:=h, \quad \theta_{4}:=h^{2} .
$$

Hence, as our model for robust control design, we consider

$$
\dot{\xi}=\tilde{A}(\theta) \xi+\tilde{B}(\theta) \omega+\tilde{B}(\theta) u
$$

where

$$
\tilde{A}(\theta)=\left[\begin{array}{cccc}
-\frac{\sigma}{m} \theta_{1}-\frac{\sigma}{J_{x x}} \theta_{1} \theta_{4} & \frac{\rho}{m} \theta_{1}+\frac{\rho}{J_{x x}} \theta_{1} \theta_{4}-\theta_{2} & -\frac{c}{J_{x x}} \theta_{3} & -\frac{k}{J_{x x}} \theta_{3}+\frac{m g}{J_{x x}} \theta_{4} \\
\frac{\rho}{J_{z z}} \theta_{1} & -\frac{\kappa}{J_{z z}} \theta_{1} & 0 & 0 \\
-\frac{\sigma}{J_{x x}} \theta_{1} \theta_{3} & \frac{\rho}{J_{x x}} \theta_{1} \theta_{3} & -\frac{c}{J_{x x}} & -\frac{k}{J_{x x}}+\frac{m g}{J_{x x}} \theta_{3} \\
0 & 0 & 1 & 0
\end{array}\right], \quad \tilde{B}(\theta)=\left[\begin{array}{c}
\frac{C_{v}}{m}+\frac{C_{v}}{J_{x x}} \theta_{4} \\
\frac{C_{v} l_{v}}{J_{z z}} \\
\frac{C_{v}}{J_{x x}} \theta_{3} \\
0
\end{array}\right]
$$

and

$$
\frac{1}{\bar{v}} \leq \theta_{1} \leq \frac{1}{\underline{v}}, \quad \underline{v} \leq \theta_{2} \leq \bar{v}, \quad \underline{h} \leq \theta_{3} \leq \bar{h}, \quad \underline{h}^{2} \leq \theta_{4} \leq \bar{h}^{2}
$$

As before, we consider PI controllers of the form

$$
\begin{aligned}
u & =K_{P} \xi+K_{I} \xi_{I} \\
\dot{\xi}_{I} & =\dot{\psi}-\alpha \delta_{d} \quad \xi_{I}(0)=0 .
\end{aligned}
$$

Recall the performance outputs $z_{1}$ and $z_{2}$ described in (29) and (30). Again, we are interested in synthesizing a stabilizing controller which minimizes the level of performance $\gamma_{1}$ for $z_{1}$ while keeping the level of performance $\gamma_{2}$ for $z_{2}$ below some prespecified level $\bar{\gamma}_{2}$. With the augmented state $x=\left[\begin{array}{ll}\xi^{T} & \xi_{I}\end{array}\right]^{T}$, the proposed controller structure can be simply described by $u=K x$ where

$$
K=\left[K_{P} K_{I}\right]
$$

and the behavior of $x$ and the performance outputs can be described by

$$
\begin{aligned}
\dot{x} & =A(\theta) x+B(\theta) \omega+B_{u}(\theta) u \\
z_{1} & =C_{1} x \\
z_{2} & =D_{2 u} u,
\end{aligned}
$$

where

$$
A(\theta)=\left[\begin{array}{cc}
\tilde{A}(\theta) & 0 \\
c_{\psi} & 0
\end{array}\right], \quad B(\theta)=\left[\begin{array}{c}
\tilde{B}(\theta) \\
-\alpha
\end{array}\right], \quad B_{u}(\theta)=\left[\begin{array}{c}
\tilde{B}(\theta) \\
0
\end{array}\right], \quad C_{1}=\left[\begin{array}{c}
\tilde{C} 0
\end{array}\right], \quad D_{2 u}=1,
$$

and $c_{\psi}=\left[\begin{array}{llll}0 & 1 & 0 & 0\end{array}\right]$. Since the matrices $A(\theta), B(\theta), B_{u}(\theta)$ depend in a multi-affine fashion on $\theta$ and each component of $\theta$ is bounded, it follows that the matrix $\left[A(\theta) B(\theta) B_{u}(\theta)\right]$ can always be expressed as a convex combination of the following 16 matrices

$$
\left[A(\theta) B(\theta) B_{u}(\theta)\right] \quad \text { where } \theta_{k} \text { equals its minimum or maximum value for } k=1, \ldots, 4 \text {. }
$$


Note here that $\theta_{k}$ denotes the $k^{\text {th }}$ element of the 4-vector $\theta$. Hence the augmented plant satisfies Assumption 4.1.

Now one can use Theorem 4.2 to design a controller which guarantees desirable output performance which is robust with respect to variations of speed and CG height which satisfy $\underline{v} \leq v \leq \bar{v}$ and $\underline{h} \leq h \leq \bar{h}$. In using Theorem 4.2 to obtain a controller which minimizes the level of performance $\gamma_{1}$ for $z_{1}$ subject to a specified level of performance $\gamma_{2}$ for $z_{2}$, we used an iterative solution algorithm similar to the one described in the Appendix.

5.2.1 Simulations. Here we present three sets of numerical simulations. The first one is the identical obstacle avoidance (elk test) scenario as in the fixed parameter case. Thus, the peak value of the driver steering input was $\delta_{p}=100^{\circ}$ and constant speed was set to be $v=140 \mathrm{~km} / \mathrm{h}$. The results are presented in Figures 5 and 6 , which demonstrate the effectiveness of the controller.
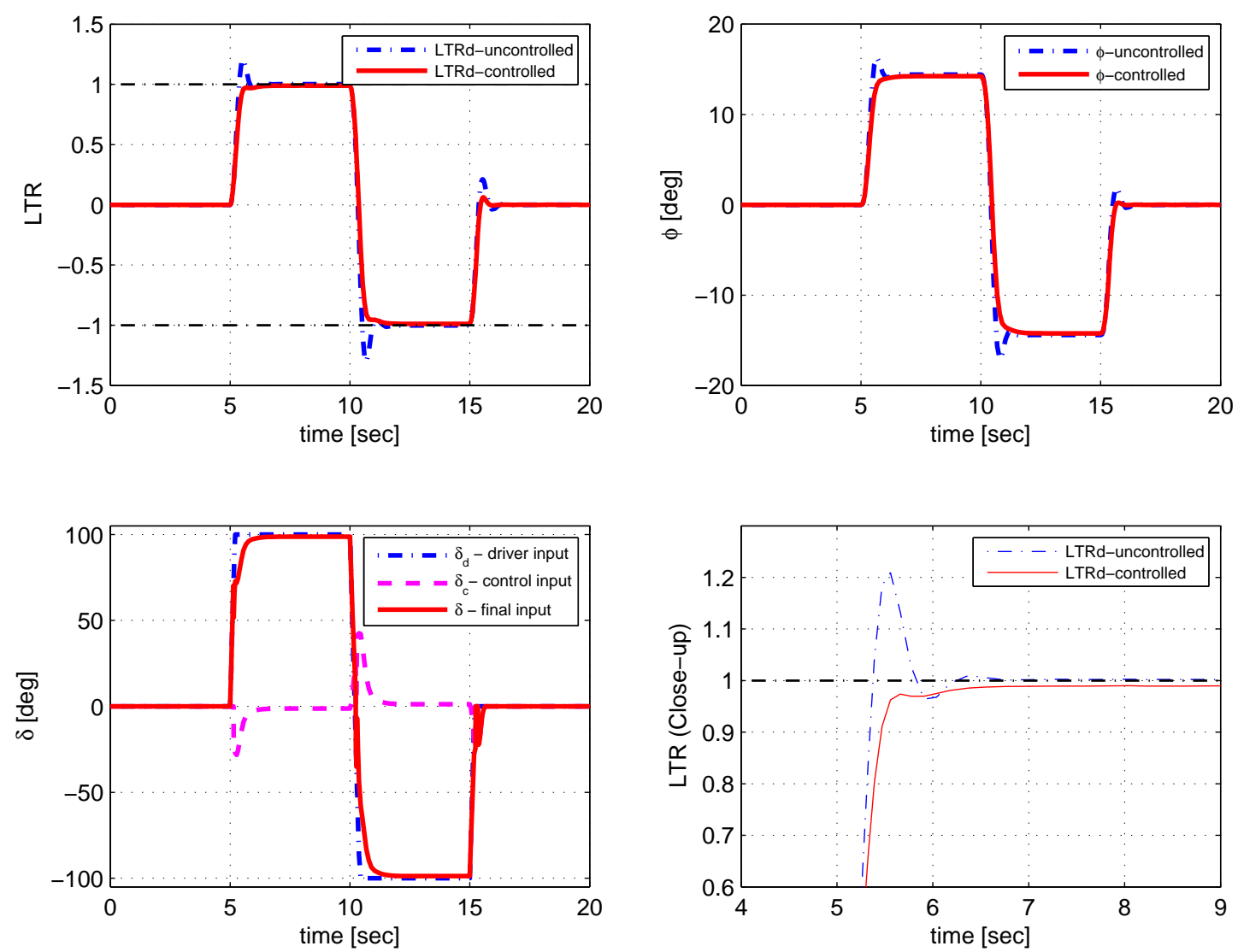

Figure 5. Comparison of the robustly controlled and the uncontrolled vehicles $\left(v=140 \mathrm{~km} / \mathrm{h}, \delta_{\text {peak }}=100^{\circ}\right.$, and $\left.h=0.375 \mathrm{~m}\right)$.

Comment : From the simulation results of the fixed and the robust controllers for the same maneuver, we observe that both methods are effective in reducing the load transfer ratio $L T R_{d}$, and thus preventing rollover. However the robust controller performance is far less conservative. Also notice that driver intervention of the controller by any chosen measure is practically undiscernible by the driver, which is favorable and was one of our aims.

In the second set of numerical simulations, we again tested a similar obstacle avoidance maneuver (elk test) however, this time we set the peak value of the driver steering input as $\delta_{p}=150^{\circ}$ and constant speed was fixed as $v=70 \mathrm{~km} / \mathrm{h}$. Moreover the CG height was selected as $h=0.45 \mathrm{~m}$. The corresponding simulation results are presented in Figures 7 and 8, which demonstrate the effectiveness of the controller for varying CG height.

In the third set of numerical simulations, we performed an obstacle avoidance maneuver with a peak driver steering input of $\delta_{p}=120^{\circ}$. Also this time we implemented a rapid change in velocity from the initial value of 

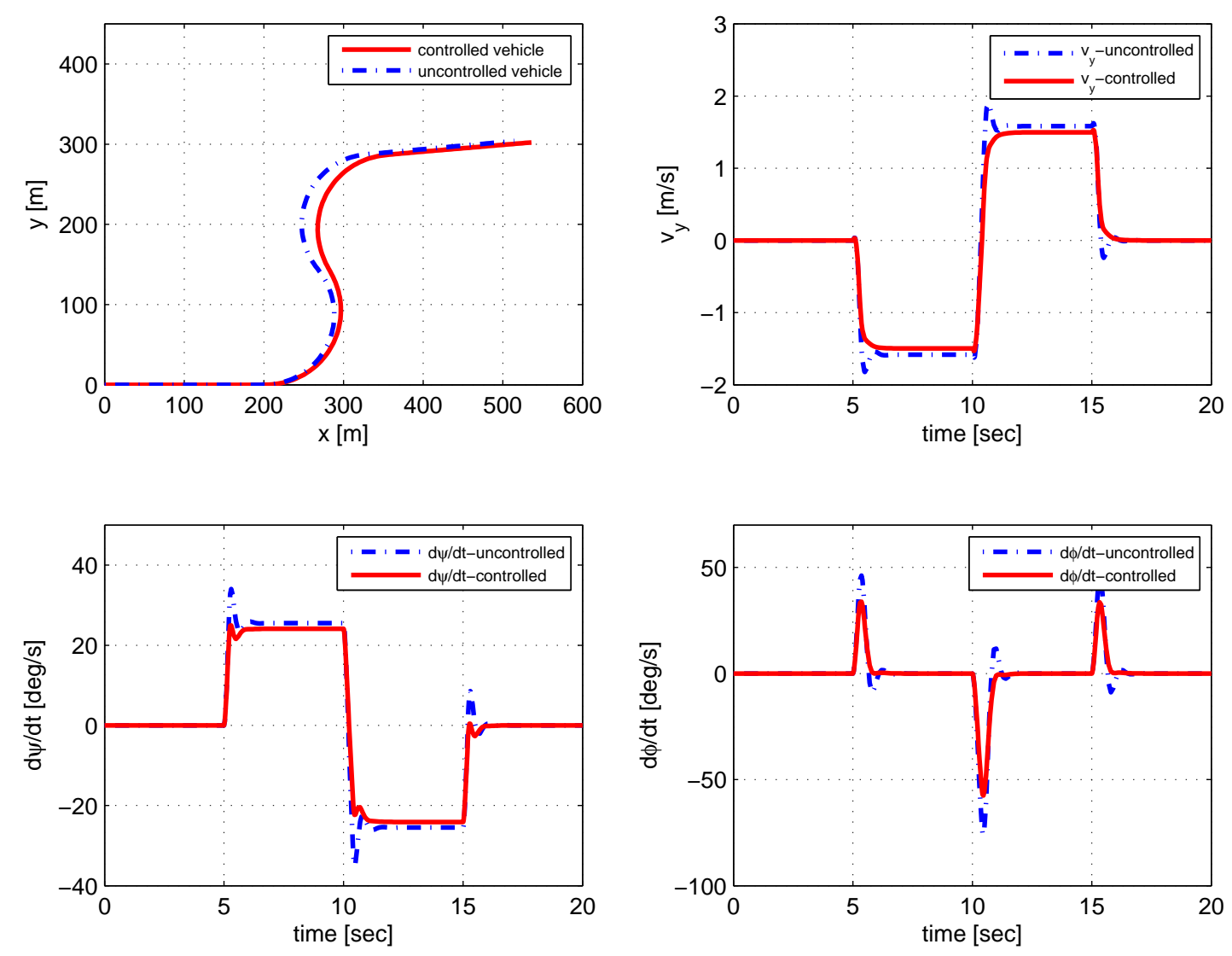

Figure 6. Comparison of the trajectories and states of robustly controlled and the uncontrolled vehicles $\left(v=140 \mathrm{~km} / h, \delta_{\text {peak }}=100^{\circ}\right.$, and $\left.h=0.375 \mathrm{~m}\right)$.

$v=140 \mathrm{~km} / \mathrm{h}$, which simulates braking action during the maneuver. In this simulation CG height was fixed to be $h=0.375 \mathrm{~m}$. The corresponding simulation results are presented in Figure 9 and Figure 10 demonstrating the effectiveness of the controller design for varying CG height and speed.

Comment : In all the simulation examples we observe that the robust controller is quite effective in reducing the load transfer ratio $L T R_{d}$ below the safety limits while keeping the controlled states to be sufficiently close to the reference vehicle states. Also notice that driver intervention of the controller is insignificant, which was one of the intended design goals.

5.2.2 Controller mode switch. A basic problem with the aforementioned controllers is that they are always active. That is, they are always attempting to limit the LTR, even in non-critical situations, thus potentially interfering with, and annoying the vehicle driver. It therefore makes sense only to activate the controller in situations where the potential for rollover is significant. Here we introduce a switching criteria for activating the controller that is based on Lyapunov theory. The reasons for considering such a switching criteria are outlined below.

The switching method introduced here is based on the Lyapunov function $V(x)=x^{T} P x$, where the positive definite symmetric matrix $P$ is given by $P=S^{-1}$ and $S$ is obtained when solving the LMIs in the controller design. Ideally, the controller is only activated when $V(x)$ reaches some critical value $V_{c r i t}$. The critical value is chosen so that $\left|L T R_{d}\right|<1$ when $V(x) \leq V_{\text {crit }}$. In particular, we regulate the controller input according to

$$
u=\left\{\begin{array}{rll}
0 & \text { if } & V(x) \leq V_{\text {crit }}-\varepsilon \\
K x & \text { if } & V(x) \geq V_{\text {crit }}
\end{array}\right.
$$



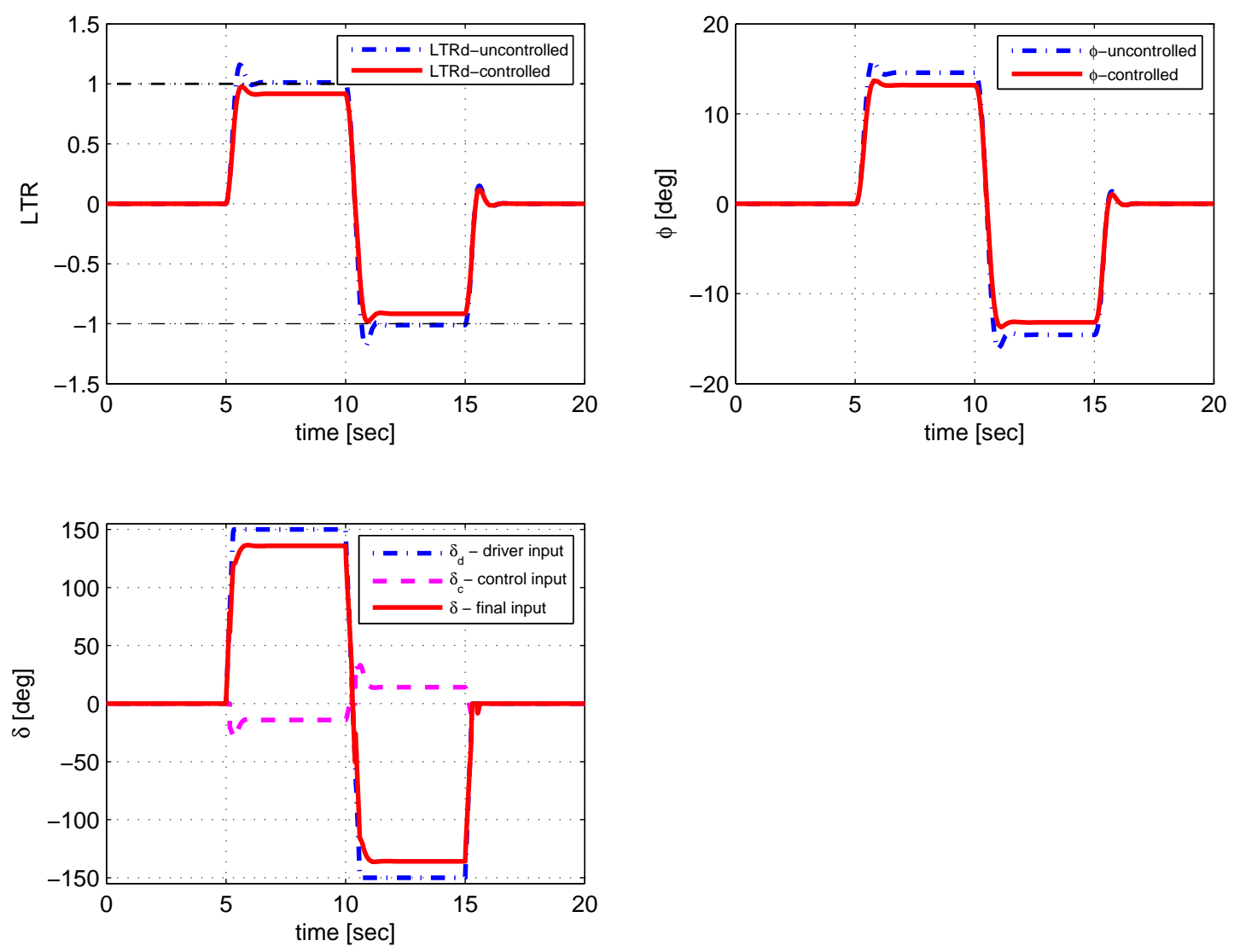

Figure 7. Comparison of the robustly controlled and the uncontrolled vehicles $\left(v=70 \mathrm{~km} / \mathrm{h}, \delta_{\text {peak }}=150^{\circ}\right.$, and $\left.h=0.45 \mathrm{~m}\right)$.

with $V_{\text {crit }}$ chosen to guarantee that the $L T R$ is close to one when the controller is activated.

The reasoning behind the above strategy is as follows. Recall from Remark 3 that our original controller design guarantees that $\dot{V}$, the time rate of change of $V$ along a solution, is negative outside the ellipsoid $\mathscr{E}\left(\rho_{\omega}\right)$ defined in (22) where $\rho_{\omega}$ is a bound on the magnitude of the disturbance input. Suppose now that the controller is not activated until $V(x)>V_{\text {crit }}$. Then for driver inputs $\omega$ which satisfy $\mu_{0}\|\omega(t)\|^{2} \leq V_{\text {crit }}$, the switching controller will guarantee that $\dot{V}$ is negative outside the ellipsoid

$$
\mathscr{E}_{\text {crit }}:=\left\{x \in \mathbb{R}^{n}: V(x) \leq V_{\text {crit }}\right\}
$$

This in turn guarantees that the ellipsoid is invariant and attractive. In particular, if a state trajectory starts at zero and $\mu_{0}\|\omega(t)\|^{2} \leq V_{\text {crit }}$ then, the state trajectory remains within this ellipsoid. Recall also that $\left\|z_{1}\right\| \leq \mu_{11} V(x)$ and $z_{1}=L T R_{d}$; hence, whenever a state trajectory starts at zero and $\mu_{0}\|\omega(t)\|^{2} \leq V_{\text {crit }}$, we have that $\left|L T R_{d}\right| \leq \mu_{11} V_{\text {crit }}$. By choosing

$$
V_{\text {crit }}<1 / \mu_{11}
$$

we guarantee that the controller turns on before $\left|L T R_{d}\right|$ reaches one, but, the controller does not switch on for small driver steering inputs. In accordance with standard practice we propose the following continuous switching-type controller to avoid chattering action:

$$
u=\zeta(V(x)) K x \quad \text { where } \quad V(x)=x^{T} S^{-1} x
$$



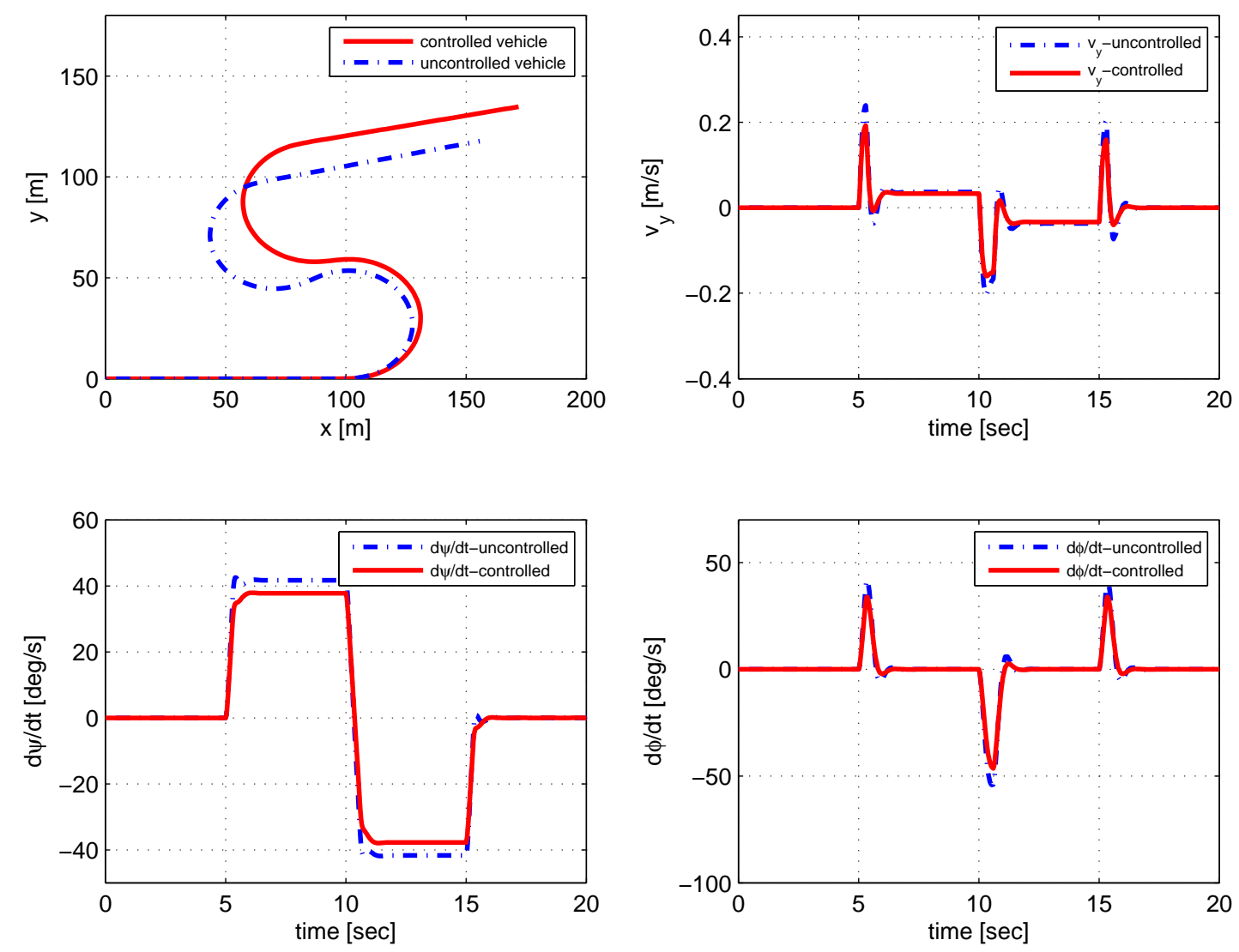

Figure 8. Comparison of the trajectories and states of robustly controlled and the uncontrolled vehicles $\left(v=70 \mathrm{~km} / \mathrm{h}, \delta_{\text {peak }}=150^{\circ}\right.$, and $\left.h=0.45 \mathrm{~m}\right)$.

and

$$
\zeta(V)=\frac{1}{2}+\frac{1}{2} s a t\left[\frac{2}{\varepsilon}\left(V-V_{c r i t}\right)+1\right]
$$

here sat denotes the saturation function and $\varepsilon$ is a small positive number. The graph of $\zeta$ is depicted in Figure 11.

We demonstrate the performance of the above switching controller with further simulations whose results are illustrated in Figure 12. These correspond to an obstacle avoidance maneuver where the peak value of the driver steering input is $\delta_{p}=50^{\circ}$ and the vehicle speed was fixed at $v=140 \mathrm{~km} / \mathrm{h}$. Notice that although there is no rollover threat in this maneuver, the original linear robust controller was trying to compensate by a very small amount as seen from the actuator input plot. Whereas the robust controller with the suggested switching produces no input and the $L T R_{d}$ corresponding to the switching controller is identical to that of the uncontrolled vehicle, demonstrating the efficacy of the suggested method.

\section{Conclusions}

We have presented a methodology for the design of vehicle rollover prevention systems using active steering actuators. By introducing the load transfer ratio $L T R_{d}$, we obtain a system performance output whose value provides an accurate measure for determining the onset of rollover. Our rollover prevention system is based upon recent results from Pancake, Corless and Brockman, which provide controllers to robustly guarantee that the peak value of the performance output of an uncertain system does not exceed a certain value. Simulation results are presented 

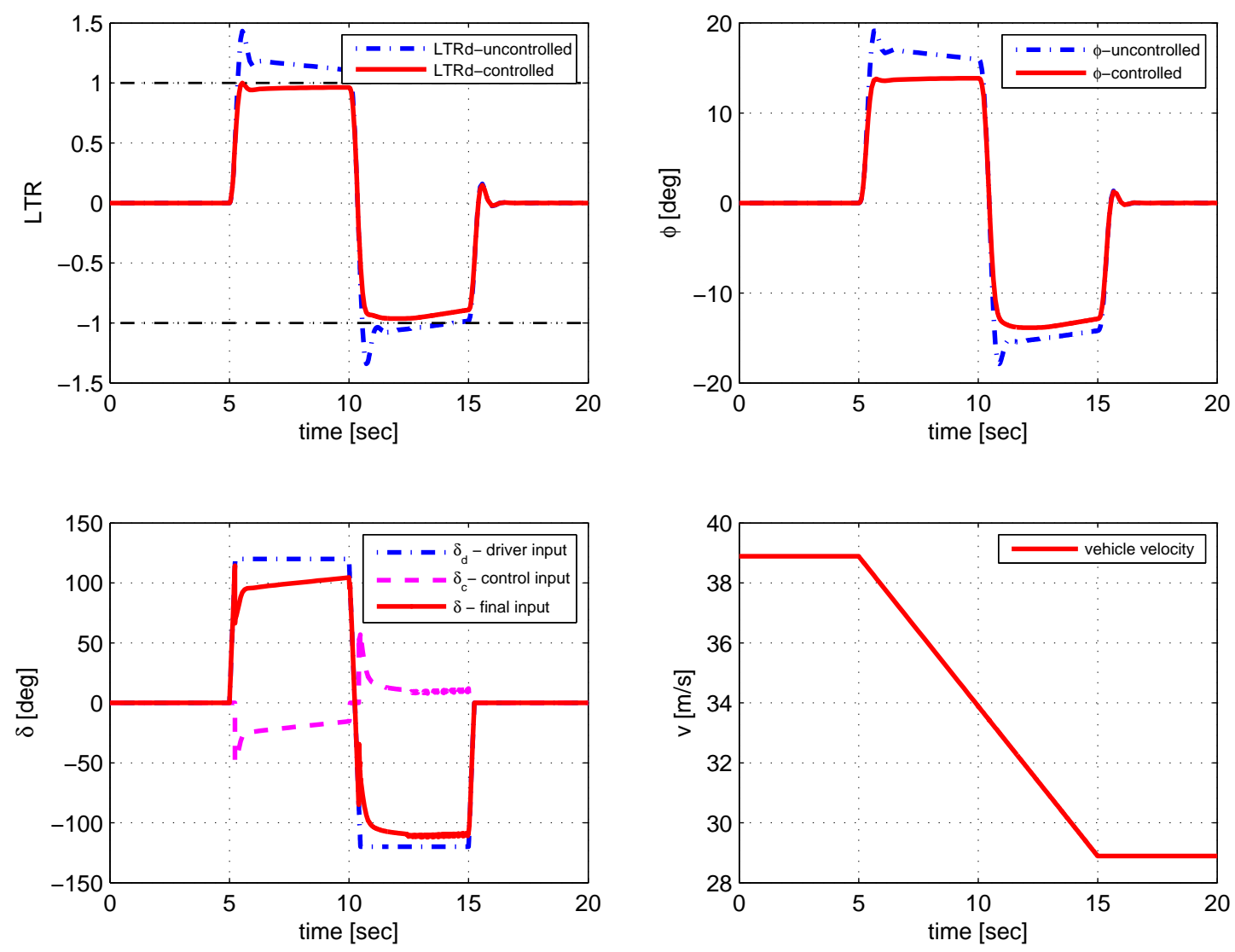

Figure 9. Comparison of the robustly controlled and the uncontrolled vehicles $\left(v_{0}=140 \mathrm{~km} / \mathrm{h}, \delta_{\text {peak }}=120^{\circ}\right.$, and $\left.h=0.375 \mathrm{~m}\right)$.

to illustrate the benefits of the proposed approach. Future work will proceed in several directions. We shall extend the methodology to include differential braking, active suspension and combinations thereof to refine our rollover prevention strategy, and analyze the resulting control allocation problem. We shall also examine the efficacy of our controllers in the presence of conditions which can result in a tripped rollover. As an alternative approach we shall also investigate a gain scheduled control approach based on locally valid fixed models and LMI based controllers as described in this paper. In another possible extension of the synthesis procedure we shall look into the use of more complex vehicle and/or tire models with the LMI algorithm.

A second strand of work will investigate refinement of the synthesis procedure. In particular, we shall investigate whether convergence and feasibility conditions can be developed to determine the existence of control gains to achieve certain pre-specified performance parameters $\gamma_{j}$.

On the practical side of this work, we have scheduled with our industrial partners an evaluation of our control design in real production vehicles. We are also looking into extending these ideas to railroad vehicles.

\section{References}

J. Ackermann and D. Odenthal, "Robust steering control for active rollover avoidance of vehicles with elevated center of gravity", Proceedings of International Conference on Advances in Vehicle Control and Safety, Amiens, France, July 1998.

S. Takano and M. Nagai, "Dynamics control of large vehicles for rollover prevention", Proceedings of the IEEE International Vehicle Electronics Conference, Tottori, Japan, September 25-28, 2001.

M. Akar, S. Solmaz and R. Shorten, "Method for Determining the Center of Gravity for an Automotive Vehicle", 

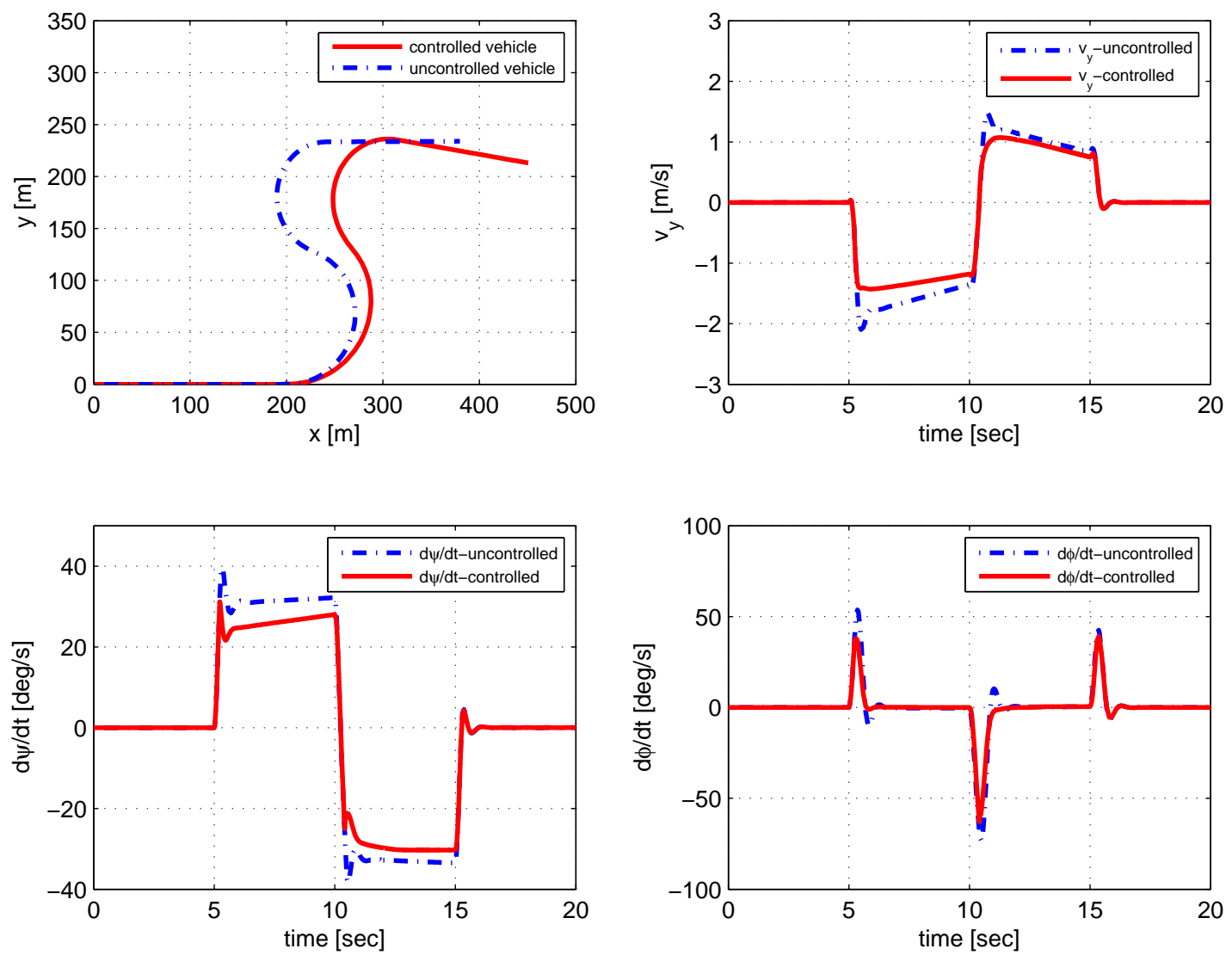

Figure 10. Comparison of the trajectories and states of robustly controlled and the uncontrolled vehicles $\left(v_{0}=140 \mathrm{~km} / \mathrm{h}, \delta_{\text {peak }}=120^{\circ}\right.$, and $\left.h=0.375 \mathrm{~m}\right)$.

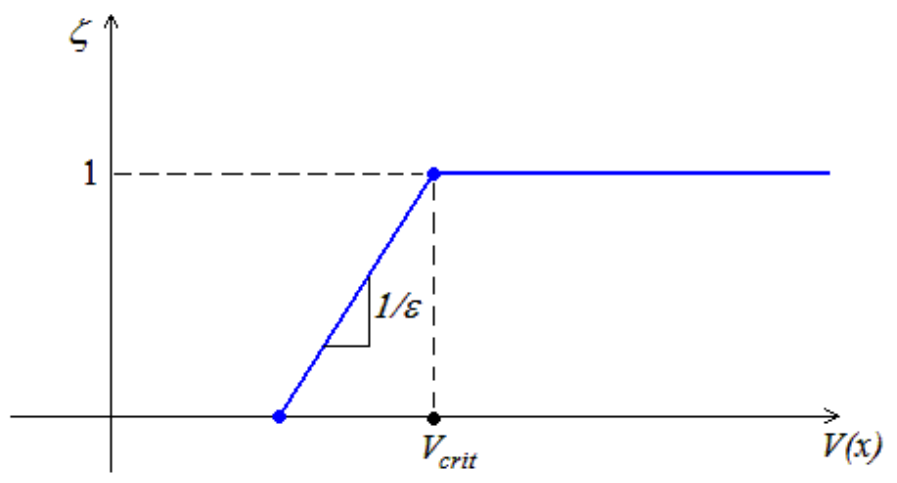

Figure 11. Graph of the function $\zeta$.

Irish Patent (2006).

C.R. Carlson and J.C. Gerdes, "Optimal rollover prevention with steer by wire and differential braking", Proceedings of ASME International Mechanical Engineering Congress and Exposition, IMECE'03, Washington, D.C., November 16-21, 2003.

B. Chen and H. Peng, "Differential-braking-based rollover prevention for sport utility vehicles with human-in-theloop evaluations", Vehicle System Dynamics, 36(4-5), pp. 359-389, 2001. 

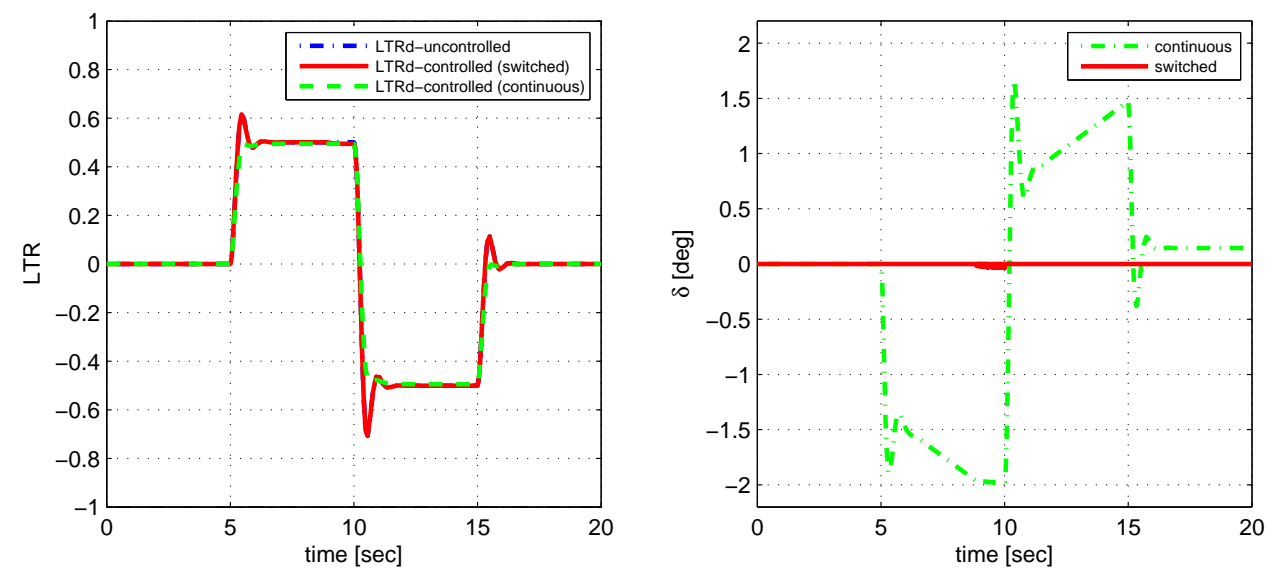

Figure 12. Comparison of continuous and switched robust controllers at a non critical maneuver $\left(v=140 \mathrm{~km} / \mathrm{h}, \delta_{\text {peak }}=50^{\circ}\right.$, and $\left.h=0.375 \mathrm{~m}\right)$.

A. Hac, T. Brown, and J. Martens, "Detection of vehicle rollover", Vehicle Dynamics \& Simulation, SAE Technical Paper Series, 2004.

R. Kamnik, F. Böttiger, K. Hunt, "Roll dynamics and lateral load transfer estimation in articulated heavyfreight vehicles: a simulation study", Proceedings of the Institution of Mechnaical Engineers, Part D, 2003.

U. Kiencke and Nielsen L., Automotive Control Systems for Engine, Driveline and Vehicle, Springer-Verlag \& SAE Int., Berlin, 2000.

D. Odenthal, T. Bünte, and J. Ackermann , "Nonlinear steering and braking control for vehicle rollover avoidance", Proceedings of European Control Conference, Karlsruhe, Germany, 1999.

L. Palkovics, À. Semsey, and E. Gerum, "Roll-over prevention system for commercial vehicles-additional sensorless function of the electronic brake system", Vehicle System Dynamics, 4, pp. 285-297, 1999.

T. Pancake, M. Corless, and M. Brockman, "Analysis and control of polytopic uncertain/nonlinear systems in the presence of bounded disturbance inputs", Proceedings of the American Control Conference, Chicago, IL, June 2000.

T. Pancake, M. Corless, and M. Brockman, "Analysis and control for a class of uncertain/nonlinear systems in the presence of bounded disturbance inputs", In preparation, 2006.

S. Solmaz, M. Corless, and R. Shorten, "A methodology for the design of robust rollover prevention controllers for automotive vehicles: Part 1-Differential Braking”, 45th IEEE Conference on Decision and Control, San Diego, USA, 2006.

S. Solmaz, M. Corless, and R. Shorten, "A methodology for the design of robust rollover prevention controllers for automotive vehicles: Part 2-Active Steering", HYCON-CEmACS Workshop on Automotive Systems and Control, Lund, June 1-2, 2006.

T.J. Wielenga, "A method for reducing on-road rollovers: anti-rollover braking", SAE Paper No. 1999-01-0123, 1999.

National Highway Traffic Safety Administration (NHTSA), "Traffic Safety Facts 2004: A Compilation of Motor Vehicle Crash Data from the Fatality Analysis Reporting System and the General Estimates System", Technical Report (2006).

\section{Appendix A: Iterative algorithm for robust control design}

In our rollover controller design we attempt to minimize the level of performance $\gamma_{1}$ while keeping the level of performance $\gamma_{2}$ below some specified level $\bar{\gamma}_{2}$. Utilizing the structure of the data in the rollover control design problem, Theorem 4.2 and Remark 2 one can solve the above minimization problem by solving the following problem: 
Minimize $\mu_{0} \mu_{11}$ subject to

$$
\begin{aligned}
{\left[\begin{array}{cc}
\beta_{i}\left(S A_{i}^{T}+A_{i} S+L^{T} B_{u_{i}}^{T}+B_{u_{i}} L\right)+S & \beta_{i} B_{i} \\
\beta_{i} B_{i}^{T} & -\mu_{0} I
\end{array}\right] \leq 0 \quad \text { for } \quad i=1, \ldots, N } \\
{\left[\begin{array}{cc}
-S & S C_{1}^{T} \\
C_{1} S & -\mu_{11} I
\end{array}\right] \leq 0 } \\
{\left[\begin{array}{cc}
-S & L^{T} \\
L & -\mu_{12} I
\end{array}\right] \leq 0 } \\
\mu_{0} \mu_{12} \leq \bar{\gamma}_{2}^{2} \\
\text { and } \\
S=S^{T}>0 \\
\mu_{0}, \mu_{11}, \mu_{12} \geq 0 \\
\beta_{i}>0 \quad \text { for } \quad i=1, \ldots, N
\end{aligned}
$$

Then $\gamma_{1}=\sqrt{\mu_{0} \mu_{11}}$ and $K=L S^{-1}$.

To solve the above optimization problem, one first needs a value of $\bar{\gamma}_{2}$ for which the above inequalities are feasible. To achieve this one can first minimize $\gamma_{2}^{2}=\mu_{0} \mu_{12}$ subject to all the inequalities above except those involving $\mu_{11}$ and $\bar{\gamma}_{2}$. After this first minimization ones obtain a value of $\gamma_{2}$ which we denote by $\gamma_{2 f}$. Now choose $\bar{\gamma}_{2} \geq \gamma_{2 f}$; in this paper, $\bar{\gamma}_{2}=5 \gamma_{2 f}$. Having obtained a feasible value of $\bar{\gamma}_{2}$, one can can then minimize $\gamma_{1}=\mu_{0} \mu_{11}$.

The above inequalities and objective functions are not linear functions of the variables. However if we separate the variables into two groups $S, L, \mu_{11}, \mu_{12}$ and $\beta_{1}, \ldots, \beta_{N}, \mu_{0}$, the inequalities are linear with respect to each group of variables. Also, we can use commercially available software to solve optimization problems with linear objective functions and linear matrix inequality constraints. Based on these observations, we propose the following iterative algorithm in an attempt to solve the above optimization problems.

Algorithm. To initiate the optimization of $\gamma_{2}$ one needs feasible symmetric matrices $S$ and $L$. These can be found by solving the corresponding quadratic stabilizability problem using the following linear matrix inequalities

$$
S A_{i}^{T}+A_{i} S+B_{u_{i}} L+L^{T} B_{u_{i}}^{T}+2 \eta S \leq 0 \quad \text { for } \quad i=1, \ldots, N
$$

for some $\eta>0$. Notice that if there is no solution to this quadratic stabilization problem, then the first inequality in (A1) does not have a solution.

The next part of the algorithm now iterates through Steps 1-3 in an attempt to minimize $\gamma_{2}$.

1. Fix $S$ and $L$ to those values obtained as a solution to (A2) or from the previous iteration.

Minimize $\mu_{0}$ subject to

$$
\begin{aligned}
& {\left[\begin{array}{rr}
\beta_{i}\left(S A_{i}^{T}+A_{i} S+L^{T} B_{u_{i}}^{T}+B_{u_{i}} L\right)+S & \beta_{i} B_{i} \\
\beta_{i} B_{i}^{T} & -\mu_{0} I
\end{array}\right] \leq 0 \quad \text { for } \quad i=1, \ldots, N} \\
& \beta_{i}>0 \text { for } i=1, \ldots, N \\
& \mu_{0} \geq 0
\end{aligned}
$$

2. Fix $\beta_{1}, \ldots, \beta_{N}$ and $\mu_{0}$ from the previous step. 
Minimize $\mu_{12}$ subject to

$$
\begin{aligned}
{\left[\begin{array}{cc}
\beta_{i}\left(S A_{i}^{T}+A_{i} S+L^{T} B_{u_{i}}^{T}+B_{u_{i}} L\right)+S & \beta_{i} B_{i} \\
\beta_{i} B_{i}^{T} & -\mu_{0} I
\end{array}\right] \leq 0 \text { for } i=1, \ldots, N } \\
{\left[\begin{array}{cc}
-S & L^{T} \\
L & -\mu_{12} I
\end{array}\right] \leq 0 } \\
S=S^{T}>0 \\
\mu_{12} \geq 0
\end{aligned}
$$

3. Let $\gamma_{2}^{2}=\mu_{0} \mu_{12}$ and return to Step 1 unless $\gamma_{2}$ has not decreased by a certain prespecified amount from the previous iteration.

Although the above steps may not achieve a global minimum for $\gamma_{2}$, a feasible value of $\gamma_{2}$ (which we denote by $\gamma_{2 f}$ ) will be obtained along with corresponding feasible $S$ and $L$ matrices. We now fix $\gamma_{2}$ at $\bar{\gamma}_{2}>\gamma_{2 f}$; in this paper, $\bar{\gamma}_{2}=5 \gamma_{2 f}$.

The next part of the algorithm attempts to minimize $\gamma_{1}$ subject to $\gamma_{2} \leq \bar{\gamma}_{2}$. It iterates through Steps 4-6.

4. Fix matrices $S$ and $L$ from the previous stage or the previous iteration.

Minimize $\mu_{0}$ subject to

$$
\begin{aligned}
{\left[\begin{array}{rl}
\beta_{i}\left(S A_{i}^{T}+A_{i} S+L^{T} B_{u_{i}}^{T}+B_{u_{i}} L\right)+S & \beta_{i} B_{i} \\
\beta_{i} B_{i}^{T} & -\mu_{0} I
\end{array}\right] \leq 0 \text { for } i=1, \ldots, N } \\
\beta_{i}>0 \text { for } i=1, \ldots, N \\
\mu_{0} \geq 0
\end{aligned}
$$

5. Fix $\beta_{1}, \ldots, \beta_{N}$ and $\mu_{0}$ from the previous step.

Minimize $\mu_{11}$ subject to

$$
\begin{aligned}
& {\left[\begin{array}{cc}
\beta_{i}\left(S A_{i}^{T}+A_{i} S+L^{T} B_{u_{i}}^{T}+B_{u_{i}} L\right)+S & \beta_{i} B_{i} \\
\beta_{i} B_{i}^{T} & -\mu_{0} I
\end{array}\right] \leq 0 \text { for } i=1, \ldots, N } \\
& {\left[\begin{array}{cc}
-S & S C_{1}^{T} \\
C_{1} S & -\mu_{11} I
\end{array}\right] \leq 0 } \\
& {\left[\begin{array}{cc}
-S & L^{T} \\
L & -\mu_{12} I
\end{array}\right] \leq 0 } \\
& \mu_{0} \mu_{12} \leq \bar{\gamma}_{2}^{2} \\
& S=S^{T}>0 \\
& \mu_{11}, \mu_{12} \geq 0
\end{aligned}
$$

6. Let $\gamma_{1}^{2}=\mu_{0} \mu_{11}$ and return to Step 4 unless $\gamma_{2}$ has not decreased by a certain prespecified amount from the previous iteration.

Note that although the iterations above may not achieve a global minimization of $\gamma_{1}$, each iteration of Steps 4-6 decreases $\gamma_{1}$. 